Journal of Mathematics and Statistics 3 (3): 88-92, 2007

ISSN 1549-3644

(C) 2007 Science Publications

\title{
Asymptotic Behavior for Solution of Reaction-Diffusion Systems
}

\author{
${ }^{1} \mathrm{M}$. Yahi and ${ }^{2} \mathrm{~K}$. Saoudi \\ ${ }^{1}$ Scientific and Technical Research Center in Welding and Testing, 16000 . Algeria \\ ${ }^{2}$ Department of Mathematics, Khenchela University, 40 000. Algeria
}

\begin{abstract}
The existence, uniqueness, and asymptotic behavior of the solution of a balanced twocomponent reaction-diffusion system have been investigated. It was shown that a global and unique solution existed and its second component can be estimated using the Lyapunov Functional. It was, also, demonstrated that each component of the solution converged, at infinity, to a constant which can be found in terms of the reacting function and the initial data. The results of the current research can be used in several areas of applied mathematics, especially when the system equations originate from mathematical models of real systems such as in Biology, Chemistry, Population Dynamics, and other disciplines.
\end{abstract}

Keywords: Reaction-Diffusion systems, Lyapunov Functional, Asymptotic Behavior, Global Existence.

\section{INTRODUCTION}

This article deals with some aspects of the diagonal reaction-diffusion system $\left(S_{1}\right)$

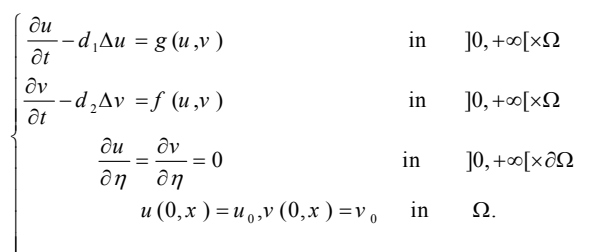

After stating local existence and uniqueness, we see some criteria for existence in the large (theorem 3.1 and 3.2) and we state the principal result (theorem 4.1) for the asymptotic behavior of the unique solution of $\left(S_{1}\right)$. This generalized results of [3], [4] and [5].

Notations and Assumptions: Therein, $\Delta=\sum_{k=1}^{n} \partial_{k}^{2}$ denotes the Laplacian operator in the Euclidian coordinates, $\Omega$ is a domain (bounded connected open set) of $\mathbb{R}^{n}$ with smooth boundary $\partial \Omega, \partial / \partial \eta$ is the outer normal on $\partial \Omega, d_{1}$ and $d_{2} \in \mathbb{R}_{+}^{*}$. Two of the most fundamental assumptions associated with $\left(\mathrm{S}_{1}\right)$ are preservation of positivity and conservation of total mass. So, we suppose that the balance condition is satisfied which meant $f(u, v)+g(u, v)=0$ and the reacting term $f$ is, also, supposed to be a nonnegative and nonlinear continuously differentiable function in $\mathbb{R}_{+}^{2}$ which verifies

$\left\{\begin{array}{c}f(0, s)=0 \quad \forall s \geq 0 \\ \lim _{s \rightarrow \infty}\left[\frac{\ln (1+f(r, s))}{s}\right] \leq \alpha^{*} \forall r \geq 0\end{array}\right.$

where

$\alpha^{*}=\frac{8 d_{1} d_{2}}{n\left\|u_{0}\right\|_{\infty}\left(d_{1}-d_{2}\right)^{2}}$.

Existence of a local solution in time: In the Banach space $C(\bar{\Omega})$ we define the norm $\|u\|_{\infty}=\sup _{x \in \bar{\Omega}}|u(x)|$. The product space $X=C(\bar{\Omega}) \times C(\bar{\Omega})$ endowed with the norm $\|(u, v)\|_{X}=\|u\|_{\infty}+\|v\|_{\infty}$ becomes a Banach space. If $w(t)=(u(t), v(t))$, then we can convert the system $\left(\mathrm{S}_{1}\right)$ to an abstract first-order system in the Banach space $X$ of the form

$\left\{\begin{array}{l}\frac{d}{d t}[w(t)]=\prod w(t)+F[w(t)], t>0 \\ w(0)=w_{0}=\left(u_{0}, v_{0}\right) \in X\end{array}\right.$

With $F[w(t)]=(-f(u, v), f(u, v))$ i.e. $F$ is a locally lipschitz function on the space $X$. The operator $\prod: D(\Delta) \times D(\Delta) \rightarrow X$ is defined by the matrix $\left(\begin{array}{cc}d_{1} \Delta & 0 \\ 0 & d_{1} \Delta\end{array}\right)$ 
where

$$
D(\Delta)=\left\{u \in C(\bar{\Omega}): \Delta u \in C(\bar{\Omega}), \frac{\partial u}{\partial \eta}=0\right\} .
$$

If $d>0$, then the operator $d \Delta$ generates an analytic semigroup of compact operators on the space $C(\bar{\Omega})$. If $S_{1}(t)$ and $S_{2}(t)$ are the analytic semigroups generated, respectively, by $d_{1} \Delta$ and $d_{2} \Delta$, on the space $C(\bar{\Omega})$, then the operator $\Pi$ generates an analytic semigroup of compact operators on the space $X$ which is given by $S(t)=\left(\begin{array}{cc}S_{1}(t) & 0 \\ 0 & S_{2}(t)\end{array}\right)$. The existence of a classical local solution of (2.1) in a maximal interval $\left[0, T_{\max }[\right.$ with initial value problem follows from standard arguments ( $c f$. Henry D. [2], Lorenzi L. and all. [7] and Pazy A. [9]).

Existence of a global solution in-time: It may be surprising that question concerning the global existence of solution to the system $\left(\mathrm{S}_{1}\right)$ remain open until recently. If $d_{1}=d_{2}$ : Nonnegative solutions of $\left(\mathrm{S}_{1}\right)$ exist globally in-time. In fact, if we add the two equations of $\left(\mathrm{S}_{1}\right)$ we get the heat equation: $\frac{\partial}{\partial t}(u+v)-d_{1} \Delta(u+v)=0$. Then, by the use of the balanced condition together with the maximum principle to obtain the $L^{\infty}$-estimation of $u$ and $v$ since $\|(u+v)(t)\|_{\infty, \Omega} \leq\left\|u_{0}+v_{0}\right\|_{\infty, \Omega}$. So, global existence of solution holds. If $d_{1} \neq d_{2}$ : The situation is quite delicate. With the homogeneous Neumann boundary conditions, Alikakos N. D. ${ }^{[1]}$ established the global existence via the embedding theorem of Sobolev and $L^{\infty}$-bounds of solutions for positive initial data when $f(u, v)=-u v^{\beta}$ and $1<\beta<\frac{n+2}{n}$. Masuda $\mathrm{K}$. ${ }^{[8]}$ Showed that the global solutions of $\left(\mathrm{S}_{1}\right)$ exist for $\beta>1$ and converge to constant when $t \rightarrow+\infty$. If the nonlinearity $f$ is polynomially bounded, then it is known that the solutions to $\left(S_{1}\right)$ exist and remain uniformly bounded in the $L^{\infty}(\Omega)$-norm. Analogous result have been also established by Haraux A. and Youkana A. ${ }^{[3]}$, and generalized their result to handle the nonlinearities of the function $f(u, v)=u \varphi(v)$ under condition $\alpha^{*}=0$, where $\varphi$ is a continuously differentiable nonnegative function not polynomially bounded but is required to grow less than exponentially.

All methods cited above (invariants regions, maximum principle, comparisons ...) have some limitations. Recall that the boundedness of the first component $u$ of the solution is guaranteed by the maximum principle which assert, in one of its version, that: If the component $u(t, x)$ verifies

$$
\left\{\begin{aligned}
\frac{\partial u}{\partial t}-d_{1} \Delta u & \leq 0 \text { in }] 0, \mathrm{~T}_{\max }[\times \Omega \\
\frac{\partial u}{\partial \eta} & \leq 0 \text { in }] 0, \mathrm{~T}_{\max }[\times \partial \Omega
\end{aligned}\right.
$$

Then $u(x, t) \leq \max _{x \in \Omega} u_{0}(x)=\left\|u_{0}\right\|_{\infty}$.

We discuss now the boundedness of the second component $v$ of the solution. A Lyapunov functional $L$ for the R-D system $\left(\mathrm{S}_{1}\right)$, is a continuously differentiable nonnegative function $L: \mathbb{R}^{+} \rightarrow \mathbb{R}^{+}$ defined by $L(t)=L(u(t, *), v(t, *))$ such that $\dot{L}(t)=\frac{\partial L[u(t, *), v(t, *)]}{\partial t} \leq 0$ for all solution $[u(t, *), v(t, *)]$ of the system $\left(\mathrm{S}_{1}\right)$.

Our main result, in this section, is:

Theorem 3.1: Suppose that $(u(t, *) ; v(t, *))$ is a solution of the system $\left(\mathrm{S}_{1}\right)$, then

$t \rightarrow L(t)=\int_{\Omega}[M-u(t, x)]^{-\gamma} \exp [\beta . v(t, x)] d x$

is a decreasing functional in the interval $\left[0, T^{*}[\right.$ iff the constants $\beta, \gamma \geq 0$ are such that

$\beta M<\gamma<\frac{4 d_{1} \cdot d_{2}}{\left(d_{1}-d_{2}\right)^{2}}$

Where $M$ verify $\left\|u_{0}\right\|_{\infty}<M$.

Proof: Differentiating the functional $L$ as a function of $t$ and using the two equations of the system $\left(\mathrm{S}_{1}\right)$ we obtain

$\dot{L}(t)=\int_{\omega} \frac{\partial}{\partial t}\left([M-u(t, x)]^{-\gamma} \exp [\beta v(t, x)]\right) d x=I+J$. where

$I=\int_{\omega}\left[\gamma d_{1}(M-u)^{-\gamma-1} e^{\beta v} \Delta u+\beta d_{2}(M-u)^{-\gamma} e^{\beta v} \Delta v\right] d x$

$J=\int_{\omega}\left[\beta(M-u)^{-\gamma}-\gamma(M-u)^{-\gamma-1}\right] . f \cdot e^{\beta v} d x$.

To compute $I$, we use a Green formula to obtain 


$$
I=-\int_{\Omega} Q(\nabla u, \nabla v)(M-u)^{-\gamma-2} e^{\beta v} d x
$$

Where

$Q(\nabla u, \nabla v)=\gamma d_{1}(\gamma+1)|\nabla u|^{2}+\beta \gamma\left(d_{1}+d_{2}\right)(M-u) \nabla u \nabla v+\beta^{2} d_{2}(M-u)^{2}|\nabla v|^{2}$.

We deduce that $Q$ is a quadratic form in $\nabla u$ and $\nabla v$ i.e. $Q(x, y)=a_{11} x^{2}+2 a_{12} x y+a_{22} y^{2}$. If $I \leq 0$ then $Q$ will be a nonnegative quadratic form all principal determinants of its symmetric matrix

$$
A_{Q}=\left(\begin{array}{cc}
d_{1} \gamma(\gamma+1) & \frac{\beta \gamma\left(d_{1}+d_{2}\right)(M-u)}{2} \\
\frac{\beta \gamma\left(d_{1}+d_{2}\right)(M-u)}{2} & \beta^{2} d_{2}(M-u)^{2}
\end{array}\right)
$$

associated to $Q$ will be nonnegative. The first principal determinant of $A_{Q}$ is $d_{1} \gamma(\gamma+1) \geq 0$, the second is $\left|A_{Q}\right|$ which is nonnegative if $\gamma \leq \frac{4 d_{1} d_{2}}{\left(d_{1}-d_{2}\right)^{2}}$.If $\quad J \leq 0 \quad$ then $\beta(M-u)^{-\gamma}-\gamma(M-u)^{-\gamma-1} \leq 0$, i.e. $\beta(M-u)-\gamma \leq 0$. Since we have $0<u<M$ for all $u$ then we obtain $\gamma>\beta M$. Thus, we have established that if $I \leq 0$ and $J \leq 0$ then (3.1) is satisfied. Now suppose that (3.1) is satisfied. First of all, recall that the Young inequality for a quadratic form, for $a \in \mathbb{R}_{+}^{*}, b$ and $c \in \mathbb{R}_{-}^{*}$, has the form $a x^{2}+b x y+c y^{2} \leq-\frac{\left(b^{2}-4 a c\right)}{2}\left[\frac{x^{2}}{4 c}+\frac{y^{2}}{4 a}\right], \quad$ for all $x, y \in \mathbb{R}$. To show that $I \leq 0$, we observe that $I=\int_{\Omega}\left[a|\nabla|^{2} b \nabla u . \nabla v+c|\nabla v|^{2}\right](M-u)^{-\gamma-2} e^{\beta v} d x$

Where

$a=-d_{1} \gamma(\gamma+1)$,

$b=-\beta \gamma\left(d_{1}+d_{2}\right)(M-u)$

and

$c=-\beta^{2} d_{2}(M-u)^{2}$.

By the Young inequality $a|\nabla|^{2}+b \nabla u \cdot \nabla v+c|\nabla v|^{2} \leq-\frac{\left(b^{2}-4 a c\right)}{2}\left[\frac{|\nabla v|^{2}}{4 a}+\frac{|\nabla u|^{2}}{4 c}\right]$.

We have $d_{1} d_{2}-\gamma\left(d_{1}-d_{2}\right)^{2}>0$ since $\gamma>\gamma / 4$. Then we obtain

$\frac{\left(b^{2}-4 a c\right)}{8 a}>\frac{d_{1} d_{2}-\gamma\left(d_{1}-d_{2}\right)^{2}}{2 d_{1}(\gamma+1)}=m_{2}>0$.

In the same way, since $0<u<M$ for all $u$ (Maximum Principle), we obtain: $\frac{\left(b^{2}-4 a c\right)}{8 c}>\frac{\gamma\left(d_{1} d_{2}-\gamma\left(d_{1}-d_{2}\right)^{2}\right)}{2 d_{2} \beta^{2} M^{2}}=m_{1}>0$.

Then

$I \leq-\int_{\Omega}\left[m_{1}|\nabla u|^{2}+m_{2}|\nabla v|^{2}\right](M-u)^{-\gamma-1} e^{\beta v} d x<0$.

To show that $J \leq 0$, use $\gamma>\beta M$ and the maximum principle to obtain $M-u \leq M$. Consequently

$J \leq-(\gamma-\beta M) \cdot M^{-(\gamma+1)} \int_{\Omega} f(u, v) \cdot e^{\beta v} d x$.

Now, let $C=(\gamma-\beta M) \cdot M^{-(\gamma+1)}$ be a nonnegative constant, then $J \leq 0$. Finally, we have showed

$\dot{L}(t) \leq-\int_{\Omega}\left[m_{1}|\nabla u|^{2}+m_{2}|\nabla v|^{2}\right](M-u)^{-\gamma-1} e^{\beta v} d x-C \int_{\Omega} f(u, v) e^{\beta v} d x \leq 0$.

\section{DISCUSSION}

We have established that $L(t) \leq k$ for some constant $k$ and hence $(M-u)^{-\gamma} e^{\beta v} \in L^{\infty}\left(\left[0, T^{*}\left[, L^{1}(\Omega)\right)\right.\right.$. Since $(M-u)^{-\gamma} \geq M^{-\gamma}$ we deduce that

$e^{\beta v} \in L^{\infty}\left(\left[0, T^{*}\left[, L^{1}(\Omega)\right)\right.\right.$

From Smoller J. [10], it is obvious that the region $E$ is invariant for the system $\left(\mathrm{S}_{1}\right)$. This ensures the stability for solution to be nonnegative for all time. Since the reaction $f$ is continuous in $\mathbb{R}_{+}^{2}$ and by the maximum principle, $0<u<M$, it follow that for all $r \in[0, M]$ we can choose $\alpha \in \mathbb{R}^{+}$to have, from (1.1), $\lim _{s \rightarrow+\infty}\left[\frac{\log (1+f(r, s))}{s}\right]<\alpha<\alpha^{*}$. Then, there is $\gamma \in \mathbb{R}^{+}$so that $f(r, s) \leq \gamma e^{\alpha s}$, for all $s \geq 0$ and $r \in[0, M]$. Now, from $\alpha<\alpha^{*}$, we have $\frac{n}{2} \alpha<\frac{4 d_{1} d_{2}}{\left\|u_{0}\right\|_{\infty}\left(d_{1}-d_{2}\right)^{2}}$. Choose $p \in \mathbb{N}_{*}^{+}$ such that $p>n / 2$ and sufficiently close to $n / 2$ such that $p \alpha<\frac{4 d_{1} d_{2}}{\left\|u_{0}\right\|_{\infty}\left(d_{1}-d_{2}\right)^{2}}$. Put $\beta=p \alpha$ to obtain $\beta\left\|u_{0}\right\|_{\infty}<\frac{4 d_{1} d_{2}}{\left(d_{1}-d_{2}\right)^{2}}$. Now, from (3.2) and since $\beta=p \alpha$ and $f(u, v) \leq \gamma e^{\alpha v}$, we deduce that:

The $p$-norm $\|f(u, v)\|_{p}$ is uniformly estimated on $\left[0, T^{*}[\right.$ for $p>n / 2$. 
Following Henry D. ${ }^{[2]}$, one has

Theorem 3.2: If $f \in C^{\infty}\left(\mathbb{R}_{+}^{2}\right)$ is a nonnegative continuously differentiable function such that $f(0, s)=0$ for all $s \geq 0$ and satisfying the condition (1.1) then all solutions of $\left(\mathrm{S}_{1}\right)$ with initial data in the region $E$, are global in time and uniformly bounded in $[0,+\infty[\times \Omega$.

Asymptotic Behavior for solution of Reactiondiffusion system: This section deals with behavior for solutions when $t \rightarrow+\infty$ of the system $\left(\mathrm{S}_{1}\right)$. With the help of boundedness of paths in the Banach space $X=C(\bar{\Omega}) \times C(\bar{\Omega})$, the asymptotic behavior for solutions can be analyzed by application of the "Invariance Principle",[6] and a result on paths precompacties which asserts that: "if $u$ and $v$ are solutions of $\left(\mathrm{S}_{1}\right)$ and if $f \in L^{\infty}([0,+\infty), C(\bar{\Omega}))$ then $u$ and $v \in L^{\infty}([0,+\infty), C(\bar{\Omega}))$ "[4].

The Main result, of this section, is the following:

Theorem 4.1: Let $(u, v)$ a global solution of the system $\left(\mathrm{S}_{1}\right)$. There is a nonnegative

constants $c_{1}$ and $c_{2}$ such that $\left\|u-c_{1}\right\|_{\infty} \stackrel{t \rightarrow \infty}{\longrightarrow} 0 \quad$ and $\quad\left\|v-c_{2}\right\|_{\infty} \stackrel{t \rightarrow \infty}{\longrightarrow} 0$.

Furthermore, we have $f\left(c_{1}, c_{2}\right)=0 \quad$ and $c_{1}+c_{2}=\frac{1}{|\Omega|} \int_{\Omega}\left(u_{0}+v_{0}\right) d x$.

To establish this result, we need the followings two lemmas.

Lemma 1: Let $\varphi \in L^{1}\left(\mathbb{R}^{+}\right)$and $\varphi(t) \geq 0$ for all $t \geq 0$. Then $\lim _{t \rightarrow+\infty} \varphi(t)=0$.

Proof: Suppose that we have $\lim _{t \rightarrow+\infty} \varphi(t)=c$ for some constant $c$. Then for all $\varepsilon>0$ there is $t_{\varepsilon}$ such that for $t>t_{\varepsilon}$ we have $\varphi(t)-c \geq-\varepsilon$. Put $\varepsilon=\frac{c}{2}, \quad$ to $\quad$ obtain $\quad \varphi(t) \geq \frac{c}{2} \quad$ and $\int_{0}^{+\infty} \varphi(t) d t \geq \int_{t_{\varepsilon}}^{+\infty} \varphi(t) d t \geq \int_{0}^{+\infty} \frac{c}{2} d t=+\infty \quad$ i.e. $\varphi \notin L^{1}\left(\mathbb{R}^{+}\right)$.

This contradicts the hypothesis.

Lemma 2: Let $K=[0,+\infty[\times \Omega$ and $(u, v)$ be a global solution of $\left(\mathrm{S}_{1}\right)$, then
$\int_{K}\left|\frac{\partial u}{\partial t}\right|^{2} d x d t<+\infty \quad$ and $\quad \int_{K}\left|\frac{\partial v}{\partial t}\right|^{2} d x d t<+\infty$.

Proof: Let $F=[0,+\infty[\times \partial \Omega$. Multiply the first equation of the system $\left(\mathrm{S}_{1}\right)$ by $\frac{\partial u}{\partial t}$ and integrate on $K$, then apply the Green formula

$\int_{K}\left|\frac{\partial u}{\partial t}\right|^{2} d x d t+d_{1} \int_{K} \nabla\left(\frac{\partial u}{\partial t}\right) \nabla u d x d t-d_{1} \int_{F}\left|\frac{\partial u}{\partial t}\right| \nabla u d x d t=-\int_{K} \frac{\partial u}{\partial t} f(u, v) d x d t$.

Then

$\int_{K}\left|\frac{\partial u}{\partial t}\right|^{2} d x d t+\frac{d_{1}}{2}\left[\int_{\Omega}|\nabla u|^{2} d x-\int_{\Omega}\left|\nabla u_{0}\right|^{2} d x\right]=-\int_{K} \frac{\partial u}{\partial t} f(u, v) d x d t$

and hence

$\int_{K}\left|\frac{\partial u}{\partial t}\right|^{2} d x d t \leq \frac{d_{1}}{2} \int_{\Omega}\left|\nabla u_{0}\right|^{2} d x+\int_{K}\left|\frac{\partial u}{\partial t} f(u, v)\right| d x d t$.

Now, apply the inequality $\frac{|k|^{2}+|h|^{2}}{2} \geq|k h|$, and put $k=\frac{\partial u}{\partial t} \quad$ and $\quad h=f(u, v), \quad$ one gets $\int_{K}\left|\frac{\partial u}{\partial t}\right|^{2} d x d t \leq d_{1} \int_{\Omega}\left|\nabla u_{0}\right|^{2} d x+\int_{K}|f(u, v)|^{2} d x d t$.

Since $u_{0} \in L^{\infty}(\Omega)$ and $f \in L^{\infty}\left(\left[0, T^{*}\right), L^{\infty}(\Omega)\right)$ we deduce $\int_{K}\left|\frac{\partial u}{\partial t}\right|^{2} d x d t<\infty$ and in the same way, we deduce that $\int_{K}\left|\frac{\partial v}{\partial t}\right|^{2} d x d t<\infty$. This prove the lemma.

According to the lemma 1, put $\varphi: t \mapsto \varphi(t)=\int_{\Omega}\left|\frac{\partial u}{\partial t}\right|^{2} d x \quad$ to obtain, by lemma 2 ,

$\lim _{t \rightarrow+\infty} \frac{\partial u(t, x)}{\partial t}=\lim _{t \rightarrow+\infty} \frac{\partial v(t, x)}{\partial t}=0$.

Now, we prove the main result of theorem 4.1. With the help of the two precedent lemmas.

Proof of theorem 4.1: The paths $\{u(t), t \geq 0\}$ and $\{v(t), t \geq 0\}$ associated to the system $\left(\mathrm{S}_{1}\right)$ are precompact in the Banach space $\mathrm{C}(\bar{\Omega}) \times \mathrm{C}(\bar{\Omega})^{[4]}$. Pick a sequence $\left(t_{n}\right)_{n \in}$ which tend to infinity when $t \rightarrow+\infty \quad$ such that $\lim _{t \rightarrow+\infty} u\left(t_{n}, *\right)=u_{1} \quad$ and 
$\lim _{t \rightarrow+\infty} v\left(t_{n}, *\right)=v_{1}$ in $\mathrm{C}(\bar{\Omega})$. Since the function $f$ is continuous, we obtain $\lim _{t \rightarrow+\infty} f\left(u\left(t_{n}, x\right)\right), v\left(\left(t_{n}, x\right)\right)=f\left(u_{1}, v_{1}\right)$

in $\mathrm{C}(\bar{\Omega})$. By (4.1), the system $\left(\mathrm{S}_{1}\right)$ becomes at the infinity, a system $\left(\mathrm{S}_{\infty}\right)$ of the form

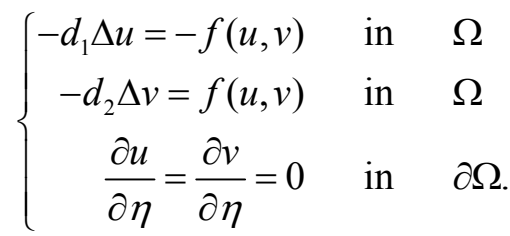

Multiply the first equation of $\left(\mathrm{S}_{\infty}\right)$ by $u_{1}$ and integrate on $\Omega$ we obtain $d_{1} \int_{\Omega} u_{1} . \Delta u_{1} d x \leq 0$. The Green Formula gives us $d_{1} \int_{\Omega}\left|\nabla u_{1}\right|^{2} d x=0$, then $\nabla u_{1}=0$ and hence $u_{1}=c_{1}$ where $c_{1}$ is a constant. On the other hand, the function $v_{1}$ verifies the homogenuous Neumann Problem

$$
\left\{\begin{array}{lll}
\Delta v_{1}=0 & \text { in } & \Omega \\
\frac{\partial v_{1}}{\partial \eta}=0 & \text { in } & \partial \Omega .
\end{array}\right.
$$

It's solution is $v_{1}=c_{2}$ where $c_{2}$ is a constant. Since the function $f$ is continuous on $\mathbb{R}_{+}^{2}$, at infinity, we obtain $f\left(c_{1}, c_{2}\right)=0$. The sequence $\left(t_{n}\right)_{n \in \mathbb{N}}$ is arbitrary, then $\left\|u-c_{1}\right\|_{\infty} \rightarrow 0$ and $\left\|v-c_{2}\right\|_{\infty} \rightarrow 0$ when $t \rightarrow+\infty$. Since the inclusion $C(\bar{\Omega}) \rightarrow L^{1}(\bar{\Omega})$ is continuous we deduce that

$$
\int_{\Omega}(u(t, x)+v(t, x)) d x \rightarrow|\Omega|\left(c_{1}+c_{2}\right)
$$

The convergence in $L^{1}(\bar{\Omega})$ is obvious since the first and the second equation of $\left(\mathrm{S}_{1}\right)$ yields $\frac{\partial}{\partial t} \int_{\Omega} u(t, x) d x \leq 0$ and $\frac{\partial}{\partial t} \int_{\Omega} v(t, x) d x \geq 0$ then $\int_{\Omega} u(t, x) d x$ is a decreasing function in $t$ and $\int_{\Omega} v(t, x) d x$ is an increasing function in $t$. But $u$ is nonnegative and $v$ is bounded, then
$\frac{1}{|\Omega|} \int_{\Omega} u(t, x) d x$ and $\frac{1}{|\Omega|} \int_{\Omega} v(t, x) d x$ converge in time and from (4.2) we deduce $c_{1}+c_{2}=\frac{1}{|\Omega|} \int_{\Omega}(u(t, x)+v(t, x)) d x=\frac{1}{|\Omega|} \int_{\Omega}\left(u_{0}+v_{0}\right) d x$.

Conclusion. The results of this study have given rise to an open similar problem of the existence, uniqueness and asymptotic behavior of the solution of general balanced $n$-diagonal (or $n$-tridiagonal) reaction-diffusion systems, which can be thought of as further research.

Acknowledgment. The author would like to thank the anonymous referee for his valuable suggestions.

\section{REFERENCES}

1. Alikakos N. D., 1979. $L^{p}$-bounds for solutions of reaction diffusion equations. Comm. Partial Differential Equations, 4: 827-868.

2. Henry D., 1981. Geometry theory of semilinear parabolic equations. Lect. Notes in Math., Springer-Verlag, New York, 840.

3. Haraux A., and Youkana A., 1988. On a result of K. Masuda concerning Reaction-Diffusion Equations. Tôhoku Math. J., 40: 159-163.

4. Kirane M., and Haraux A., 1983. Estimation $C^{1}$ pour des problèmes paraboliques semilineaires. Annal. Fac. des Sc. Toulouse, 5: 265280.

5. Kouachi S., and Youkana A., 2001. Global existence for a class of reaction-diffusion systems. Bull. of Polish Academy of Sciences, 49 No 3: 303-308

6. La Salle J. P., 1976. Stability theory and invariance principle. Dynamical systems, An International Symposium. Academic Press, New-York, Vol. I: 211-222

7. Lorenzi L., Lunardi A., Metafune G. and Diego P., 2005. Analytic Semigroups and ReactionDiffusion Problems. Internet Seminar: 1-108.

8. Masuda K., 1983. On Global existence and Asymptotic behavior of solutions of reactiondiffusion equations. Hokkaido Math. J., 12: 360-370

9. Pazy A., 1983. Semigroups of Linear Operators and Applications to Partial Differential Equations. Springer-Verlag, New York.

10. Smoller J., 1983. Shock Waves and ReactionDiffusion Equations. Springer-Verlag, Berlin. 